\title{
RIGIDITY AND EXPANSION FOR RATIONAL MAPS
}

\author{
PETER HAÏSSINSKY
}

\begin{abstract}
A general approach is proposed to prove that the combination of expansion with bounded distortion yields strong rigidity of conjugacies.
\end{abstract}

\section{Introduction}

We present results on the rigidity of rational maps which satisfy some expansion properties. These results are based on D. Sullivan's ideas on hyperbolic maps: we use expansion and bounded distortion properties to derive statements on rigidity. This work is also inspired by F. Przytycki and S. Rohde's work on topological Collet-Eckmann maps (see [20]) and by results on invariant line fields due to C. McMullen (see [13]).

Let $f: \overline{\mathbb{C}} \longrightarrow \overline{\mathbb{C}}$ be a rational map of degree $d \geqslant 2$. Let us recall that its Julia set $J(f)$ is the set of points such that the family of iterates of $f$ is not normal in any neighbourhood. For any $z \in J(f), \delta \geqslant 1$ and $r>0$, we are interested in the set of iterates such that a small neighbourhood of $z$ is mapped by a degree $\delta$ map onto a set of definite size, that is a ball of radius $r$. More precisely, as in [20], let us define

$$
E(z, \delta, r)=\left\{n \geqslant 1, \operatorname{deg}\left(f^{n}: B_{n}(z) \longrightarrow D\left(f^{n}(z), r\right)\right) \leqslant \delta\right\},
$$

where $B_{n}(z)$ is the simply connected component of $f^{-n}\left(D\left(f^{n}(z), r\right)\right)$ containing $z$ when it exists. Given $r$ and $\delta$ as above, we define $G(\delta, r)$ to be the set of points $z \in J(f)$ such that $E(z, \delta, r)$ is infinite.

DeFINITION 0.1. We say that a rational map $f$ of degree $d \geqslant 2$ is weakly hyperbolic if $J(f) \backslash \bigcup_{r, \delta} G(\delta, r)$ has Lebesgue measure zero. We say that $f$ is uniformly weakly hyperbolic if there exists $\left(\delta_{0}, r_{0}\right)$ such that $J(f) \backslash G\left(\delta_{0}, r_{0}\right)$ has at most $\sigma$-finite length.

THEOREM 0.2. Let $f$ be a rational map of degree $d \geqslant 2$.

(i) If $f$ is weakly hyperbolic and if its Julia set carries an invariant line field, then $f$ is a Lattès example.

(ii) If $f$ is uniformly weakly hyperbolic and is conjugate to another rational map $g$ by a homeomorphism $\varphi: \overline{\mathbb{C}} \longrightarrow \overline{\mathbb{C}}$, quasiconformal outside $J(f)$, then $\varphi$ is actually globally quasiconformal. In particular, if $J(f)=\overline{\mathbb{C}}$ and if $f$ is not a Lattès example, then $\varphi$ is a Moebius map.

Background literature on quasiconformal maps can be found in [1]. From Theorem 0.2 , we deduce the following corollary.

Received 5 October 1999; revised 9 February 2000.

2000 Mathematics Subject Classification 30D05, 37Fxx, 30C62.

This work was supported by Finnish Academy of Science grant 42948.

J. London Math. Soc. (2) 63 (2001) 128-140. (C) London Mathematical Society 2001. 
COROLLARY 0.3. If $f$ is uniformly weakly hyperbolic and is topologically conjugate to another rational map by a homeomorphism $\varphi$, then $\varphi$ is isotopic rel $J(f)$ to a quasiconformal conjugacy.

These results have applications to pinching deformations and geometric matings which motivated this work (see $[6,7]$ ); it follows from Theorem 0.2 that geometric matings of uniformly weakly hyperbolic polynomials with locally connected Julia sets are well defined up to conformal conjugacy, when they are known to exist. Moreover, when equicontinuity of a pinching deformation of such maps prevails, then it implies convergence of this deformation (unique limit).

In this paper, we first review bounded distortion lemmas that represent the main tools for our study $(\S 1)$. In $\S 2$, we relate our growth condition to Zalcman's lemma. In the next section, we give examples of uniformly weakly hyperbolic maps. Then, in $\S 4$, the first results on rigidity are obtained by showing that, for weakly hyperbolic maps, either the Julia set is the whole sphere or its Lebesgue measure is vanishing. We use the fact that we can blow up small scales to definite size to show that positive measure of the Julia set implies that it is the whole sphere. In $\S 5$, we prove that the Julia sets of weakly hyperbolic maps do not carry any invariant line fields provided they are not Lattès examples. To do so, we use the same ideas as in the previous section. Then, for uniformly weakly hyperbolic maps, we show that topological conjugacies which are quasiconformal off the Julia set are actually globally quasiconformal. In this case, we proceed the other way around; we use our knowledge of the geometry at large scales to deduce infinitesimal properties. From the preceding result on invariant line fields, we deduce Theorem 0.2 . We then deal with the corollary. In the Appendix, we show that, even though parabolic points are not 'good points' for expansion, they still satisfy some nice properties with respect to conjugacies.

For related results on rigidity statements, one can consult $[\mathbf{5}, \mathbf{1 2}, \mathbf{1 5}, \mathbf{1 7}, \mathbf{2 0}, \mathbf{2 1}, \mathbf{2 3}]$.

Notation 0.4 . The unit disk will be denoted by $\mathbb{D}$. For $a, b>0$, we will write $a \asymp b$ if there is an absolute constant $u>1$, independent of $a$ and $b$, such that $1 / u \leqslant$ $(a / b) \leqslant u$.

\section{Distortion bounds for finite degree maps}

Here, we review distortion lemmas that we will need throughout the proof of Theorem 0.2. For $x \in \mathbb{C}$ and $r>0$, we denote by $D(x, r)$ the Euclidean disk centred at $x$ of radius $r$.

Let us recall that a proper map $f: U \longrightarrow V$ is a map such that any compact subset of $V$ has a compact preimage in $U$. Therefore, $f$ has to be onto and if $f$ is also holomorphic then, by the argument principle, every point in $V$ has a constant number of preimages counted with multiplicity which we define as the degree of $f$.

We start with two lemmas which estimate the modulus of an annulus (the first is classic, see for instance [13, Theorem 2.4]).

Lemma 1.1. There exists $1>r(m)>0$ such that, if $K \subset \mathbb{D}$ is a compact set containing 0 and if $\mathbb{D} \backslash K$ is an annulus of modulus greater than $m>0$, then $K \subset$ $D(0, r(m))$. 
Lemma 1.2. For any $m>0$ and any integer $d \geqslant 1$, there is a constant $C_{0}=$ $C_{0}(m, d)>0$ such that if $B: \mathbb{D} \longrightarrow \mathbb{D}$ is a holomorphic proper map of degree $d$ fixing 0 and if $K$ is a full compact subset of $\mathbb{D}$ containing 0 with $\bmod (\mathbb{D} \backslash K) \geqslant m$, then, for the connected component $K^{\prime}$ of $f^{-1}(K)$ containing the origin,

$$
\bmod \left(\mathbb{D} \backslash K^{\prime}\right) \geqslant C_{0} .
$$

Proof. Let $D$ be the disk of radius $r(m)$ centred at the origin given by Lemma 1.1. Denote by $D^{\prime}$ the connected component of $B^{-1}(D)$ containing the origin; the maximum principle implies that $D^{\prime}$ is simply connected. For each critical value $v$ outside $D$, consider the circle $S_{v}=\{z:|z|=|v|\}$. The union of these circles splits $\mathbb{D} \backslash D$ into a finite number of annuli $A_{i}$. Every connected component of $f^{-1}\left(A_{i}\right)$ is also an annulus of modulus at least $\bmod A_{i} / d$, so by Grötzsch inequality, we obtain

$$
\bmod \left(\mathbb{D} \backslash K^{\prime}\right) \geqslant \bmod (\mathbb{D} \backslash \bar{D}) \geqslant(1 / 2 \pi d) \cdot \log (1 / r(m)) .
$$

Hence, the lemma is proved with $C_{0}=(1 / 2 \pi d) \cdot \log (1 / r(m))$.

Definition 1.3. Let $U \subset \mathbb{C}$ be a bounded and simply connected domain of the plane. For $x \in U$, we define the roundness of $U$ with respect to $x$ by

$$
\operatorname{Round}(U, x)=\inf \{R / r, D(x, r) \subset U \subset D(x, R)\} .
$$

The following theorem is to be compared with similar results given for example in $[\mathbf{1 8}, \mathbf{1 9}, \mathbf{2 5}]$. For a more classical reference, see $[\mathbf{8}]$.

THEOREM 1.4 (bounded distortion). For any $m>0$ and $d \in \mathbb{N} \backslash\{0\}$, there exists $C=C(m, d) \geqslant 1$ such that the following is true. Let $f: U \longrightarrow V$ be a holomorphic proper map of degree d between bounded simply connected sets of the plane, and let $D \subset V$ be simply connected with compact closure in $V$ and with $\bmod (V \backslash \bar{D}) \geqslant m$; fix $z \in D$ and assume that $\operatorname{Round}(D, z) \leqslant K$. If $D^{\prime}$ is a simply connected component of $f^{-1}(D)$ and $w \in D^{\prime} \cap f^{-1}(z)$ then $\operatorname{Round}\left(D^{\prime}, w\right) \leqslant C \cdot K$.

This theorem follows from the following lemmas.

Lemma 1.5 (Koebe). For any $m>0$, there exists $C_{1}=C_{1}(m)>1$ such that the following is satisfied. Let $f: U \longrightarrow V$ be a conformal map between bounded simply connected sets of the plane, and let $D \subset U$ be simply connected with compact closure in $U$ and with $\bmod (U \backslash \bar{D}) \geqslant m$; for $z \in D$, $\operatorname{Round}(f(D), f(z)) \leqslant C_{1} \cdot \operatorname{Round}(D, z)$.

Moreover, $C_{1}(m) \rightarrow 1$ when $m \rightarrow \infty$.

Proof. From [13, Theorem 2.9], there exists $C_{1}(m)$ such that, for any $z, w_{1}$ and $w_{2}$ in $D$,

$$
\left(1 / C_{1}\right) \cdot \frac{\left|z-w_{1}\right|}{\left|z-w_{2}\right|} \leqslant \frac{\left|f(z)-f\left(w_{1}\right)\right|}{\left|f(z)-f\left(w_{2}\right)\right|} \leqslant C_{1} \cdot \frac{\left|z-w_{1}\right|}{\left|z-w_{2}\right|} .
$$

The roundness corresponds to an extremal case, so the lemma follows.

LemMa 1.6. For any integer $d \geqslant 1$, there is a constant $C_{2}=C_{2}(d)>0$ such that the following is true. Let $B: \mathbb{D} \longrightarrow \mathbb{D}$ be a holomorphic proper map of degree $d \geqslant 1$ such that $B(0)=0$, and let $\Omega \subset \mathbb{D}$ be a simply connected set containing the origin with $\operatorname{Round}(\Omega, 0) \leqslant K$. 
Denote by $\Omega^{\prime}$ the connected component of $B^{-1}(\Omega)$ containing the origin; then

$$
\operatorname{Round}\left(\Omega^{\prime}, 0\right) \leqslant C_{2} \cdot K \text {. }
$$

Proof. Let $t=\sup _{\Omega}|z|$. By definition, $D(0, t / K) \subset \Omega$ and it follows from the Schwarz lemma that $|B(z)| \leqslant|z|$; hence $D(0, t / K) \subset \Omega^{\prime}$.

If $t \geqslant(1 / 2)$, then one can also write $\sup _{\Omega^{\prime}}|z| \leqslant 1 \leqslant 2 t$, so that $D(0, t / K) \subset \Omega^{\prime} \subset$ $D(0,2 t)$ and $\operatorname{Round}\left(\Omega^{\prime}, 0\right) \leqslant 2 \cdot K$.

If $t<(1 / 2)$, let $D$ be the connected component of $B^{-1}(D(0,2 t))$ containing the origin. Note that $D$ is simply connected by the maximum principle. Let $h_{t}: \mathbb{D} \longrightarrow D$ be a conformal map such that $h_{t}(0)=0$ and define for $z \in \mathbb{D}$ the map $\hat{B}(z)=$ $(1 / 2 t) \cdot B \circ h_{t}(z)$ : this is a holomorphic proper map of degree at most $d$ onto $\mathbb{D}$.

Now we can apply the preceding result and deduce that

$$
\operatorname{Round}\left(h_{t}^{-1}\left(\Omega^{\prime}\right), 0\right) \leqslant 2 \cdot K \text {. }
$$

Moreover, $\bmod \left(\mathbb{D} \backslash h_{t}^{-1}\left(\Omega^{\prime}\right)\right) \geqslant C_{0}(\log 2, d)$ by Lemma 1.2 ; so, Lemma 1.5 yields

$$
\operatorname{Round}\left(\Omega^{\prime}, 0\right) \leqslant 2 \cdot C_{1}\left(C_{0}(\log 2, d)\right) \cdot K \text {. }
$$

Therefore, the lemma is proved with $C_{2}=2 \cdot C_{1}\left(C_{0}(\log 2, d)\right)$.

Proof of Theorem 1.4. Let $h_{U}: \mathbb{D} \longrightarrow U$ (respectively $h_{V}: \mathbb{D} \longrightarrow V$ ) be a conformal map such that $h_{U}(0)=w$ (respectively $\left.h_{V}(0)=z\right)$. Set $W=h_{V}^{-1}(D)$. Lemma 1.5 implies that $W$ has roundness with respect to 0 less than $C_{1}(m) \cdot K$. Furthermore, from Lemma 1.6, Round $\left(W^{\prime}, 0\right) \leqslant C^{\prime} \cdot K$, where $C^{\prime}$ depends only on $m$ and $d$. Finally, another application of Lemma 1.5 yields

$$
\operatorname{Round}\left(D^{\prime}, w\right) \leqslant C^{\prime} \cdot C_{1}(m / d) \cdot K \text {. }
$$

Corollary 1.7. For any $m>0, K, \kappa \geqslant 1$ and $d \in \mathbb{N} \backslash\{0\}$, there exists $C^{\prime}=$ $C^{\prime}(m, K, \kappa, d) \geqslant 1$ such that the following is true. Let $f: U \longrightarrow V$ be a $\kappa$-quasiregular mapping of degree d between bounded simply connected sets of the plane, and let $D \subset V$ be simply connected with compact closure in $V$ and satisfying $\bmod (V \backslash \bar{D}) \geqslant m$; fix $z \in D$ and assume that $\operatorname{Round}(D, z) \leqslant K$. If $D^{\prime}$ is a simply connected component of $f^{-1}(D)$ and if $w \in D^{\prime} \cap f^{-1}(z)$ then $\operatorname{Round}\left(D^{\prime}, w\right) \leqslant C^{\prime}$.

Proof. Such a map $f$ admits Stoilow factorization: it can be written as the composition of a holomorphic degree $d$ map with a quasiconformal map. However, in $\mathbb{C}$, quasiconformal maps are also quasisymmetric; hence, by definition, they distort roundness by a factor which only depends on its quasisymmetric characteristics. Thus, the corollary follows from Theorem 1.4.

REMARK 1.8. Theorem 1.4 and Corollary 1.7 remain true when stated with the spherical metric if we assume that $\overline{\mathbb{C}} \backslash V$ contains a ball of spherical radius bounded from below by an $\varepsilon>0$, which will be the case in our applications. The constants $C$ and $C^{\prime}$ will then also depend on $\varepsilon$.

\section{Good sets and Zalcman's lemma}

While the author was preparing this paper, K. Astala remarked that the sets $G(\delta, r)$ are closely related to Zalcman's lemma (see [27] and references therein). $\mathrm{He}$ has kindly accepted that his remark be included here. 
Let us first state Zalcman's lemma.

THEOREM 2.1 (L. Zalcman). Let $\left(f_{n}\right)_{n}$ be a family of meromorphic maps defined in the unit disk. This sequence is not normal at the origin if and only if there are sequences $z_{k} \rightarrow 0, \quad n_{k} \rightarrow \infty$ and positive $\rho_{k} \rightarrow 0$ such that $g_{k}(z)=f_{n_{k}}\left(z_{k}+\rho_{k} z\right)$ is uniformly convergent on compact subsets to an open and meromorphic function $g: \mathbb{C} \longrightarrow \overline{\mathbb{C}}$ with spherical derivative 1 at the origin.

Remark 2.2. This result has been recently applied to complex dynamics to provide a very short proof that repelling points are dense in Julia sets of rational maps and meromorphic functions (see $[\mathbf{2}, \mathbf{3}, \mathbf{2 2}]$ ).

This criterion implies that, given a rational map $f$ and any point $\zeta \in J(f)$, there are renormalized subsequences $g_{k}$ of $f^{n_{k}}$ uniformly converging to an open map $g$.

Proposition 2.3 (jointly with K. Astala). With the notations from above, if $\sup \left|z_{k}-\zeta\right| / \rho_{k}<\infty$ then $\zeta \in G(\delta, r)$ for some $(\delta, r)$.

Conversely, if $\zeta \in G(\delta, r)$, then there are sequences $n_{k} \rightarrow \infty$ and positive $\rho_{k} \rightarrow 0$ such that $\left(f^{n_{k}}\left(\zeta+\rho_{k} z\right)\right)_{k}$ forms a normal family defined on a disk, and all the limits are open mappings. Moreover, one can choose $z_{k} \rightarrow \zeta$ so that $\sup \left|z_{k}-\zeta\right| / \rho_{k}<\infty$ and the limit function has non-zero derivative at the origin.

Remark 2.4. Whether or not the sequence $\left(g_{k}\right)$ we obtain in Proposition 2.3 is normal on the whole plane is not clear. For example, let $P$ be a polynomial with a Siegel disk centred at the origin, and define $f_{n}(z)=P^{n}(n z)$. Then this sequence is not normal at the origin, and if we set $\rho_{n}=1 / n$, then we get a normal family in a neighbourhood of the origin, but not on the whole plane.

Proof of Proposition 2.3. Without loss of generality, we will assume that $\zeta=0$.

Suppose that $\sup \left|z_{k}\right| / \rho_{k}<\infty$. Without loss of generality, we may assume that $-z_{k} / \rho_{k} \rightarrow \hat{z}$. Fix any $r>0$, and let $B^{\prime}$ be the connected component of $g^{-1}(D(g(\hat{z}), 2 r))$. If $r$ is small enough, then $B^{\prime}$ is bounded and the restriction of $g$ to $B^{\prime}$ has a finite degree $\delta$. When $k$ is large enough, $g_{k}$ also has finite degree on $B^{\prime}$ by Rouché's theorem. Since $g_{k}\left(-z_{k} / \rho_{k}\right)=f^{n_{k}}(0)$, there is a neighbourhood of the origin $B_{k}$ such that $f^{n_{k}}\left(B_{k}\right)=$ $D\left(f^{n_{k}}(0), r\right)$, and the degree of this map is at most $\delta$. This implies that $0 \in G(\delta, r)$.

Let us now assume that $0 \in G(\delta, r)$ for some $(\delta, r)$. Lemma 2.5 below implies that, for $n \in E(z, \delta, r)$, there are positive $\rho_{n} \rightarrow 0$ such that

$$
D\left(f^{n}(0), c \cdot r\right) \subset f^{n}\left(D\left(0, \rho_{n}\right)\right) \subset D\left(f^{n}(0), r\right),
$$

where $c>0$ is a constant independent of $n$. Therefore, $z \longmapsto f^{n}\left(z \cdot \rho_{n}\right)$ is a normal family and any limit map $g$ is open. If ever $g^{\prime}(0)=0$, then choose another point $z_{0}$ with non-zero derivative. Then, $g_{n}(z)=f^{n}\left(\left(z_{0}+z\right) \cdot \rho_{n}\right)$ is also a normal family and the limit has a non-vanishing derivative at the origin. By definition, $\sup \left|z_{n}\right| / \rho_{n}=$ $\left|z_{0}\right|<\infty$.

LEMMA 2.5. Let $f: W \longrightarrow \mathbb{D}$ be a holomorphic proper map of degree $d \geqslant 1$, where $d(0, \partial W)=1$ and such that $f(0)=0$. Then, there is $c=c(d)>0$ such that $f(\mathbb{D})$ contains the disk $D_{c}$ of radius $c$ centred at 0 . 
Proof. Let $D_{c}$ be the maximal disk of radius $c$ centred at 0 such that $D_{c} \subset f(\mathbb{D})$ and denote by $D_{c}^{\prime}$ the connected component of $f^{-1}\left(D_{c}\right)$ containing 0 . By Lemma 1.2, we get $\bmod \left(W \backslash \bar{D}_{c}^{\prime}\right) \geqslant(1 / 2 \pi d) \log (1 / c)$.

On the other hand, $\left(W \backslash \bar{D}_{c}^{\prime}\right)$ is an annulus which separates 0 from $\infty$ such that both boundary components intersect the unit circle; its modulus is bounded from above by an absolute constant.

Gluing together both estimates on the modulus yields a positive lower bound for $c$.

In a forthcoming paper, K. Astala and this author will give an improved picture of the links between the growth of $\left|z_{k}\right| / \rho_{k}$ and the behaviour of the iterates of rational maps.

\section{Examples}

We start by giving examples of uniformly weakly hyperbolic maps. Unfortunately, the author does not know of any sharp example. All the ones given below satisfy better properties.

The most obvious examples are provided by hyperbolic maps, that is for which there exists $r>0$ such that $J(f)=G(1, r)$. More involved maps consist of so-called 'semi-hyperbolic maps' [4], introduced by L. Carleson, P. Jones and J. C. Yoccoz in the setting of polynomials which satisfy $E(z, \delta, r)=\mathbb{N}$ for some $(\delta, r)$ and all $z \in J(f)$ (see also [12]). Along the same trend of ideas, there are 'topological Collet-Eckmann maps' introduced by F. Przytycki and S. Rohde; for these maps, $\inf (E(x, \delta, r) \cap$ $[1, n] / n) \geqslant 1 / 2$ for every $x \in J(f)$ and for some fixed $(\delta, r)$. These maps include all the preceding examples and also Collet-Eckmann maps for which the Lyapunov exponents at the critical values are strictly positive (see $[19,20])$. For all these maps, the exceptional set is empty.

There are also rational maps which satisfy a summability condition, namely, there exists $\alpha>0$ such that, for all critical (not postcritical) points $c$ of the Julia set,

$$
\sum_{n \geqslant 0}\left|\left(f^{n}\right)^{\prime}(f(c))\right|^{-\alpha}<\infty .
$$

J. Graczyk and S. Smirnov have proved that rational mappings without any parabolic points satisfying the above condition with $\alpha<\left(1 /\left(1+d_{c}\right)\right)$, where $d_{c}$ is the maximal multiplicity of the critical points on the Julia set of $f^{n}$ for all $n \geqslant 1$, are uniformly weakly hyperbolic. Moreover, the dimension of the exceptional set is strictly smaller than one. It would also seem possible to construct examples with exceptional sets of positive dimension (see [5]).

Another type of example (which was at first the main motivation for this work) consists of rational maps with no recurrent critical point on their Julia set. We denote them by $\mathrm{NR}(d)$. These maps are called geometrically finite by M. Lyubich.

Proposition 3.1. Any map of $\mathrm{NR}(d)$ is uniformly weakly hyperbolic.

We first recall a theorem due to R. Mañé that we will use to prove Proposition 3.1, in order to define 'good' coverings for our purpose. 
THEOREM 3.2 (R. Mañé $[\mathbf{1 6}, \mathbf{2 5}])$. Let $f$ be a rational map. If a point $x \in J(f)$ is not a parabolic periodic point and is not contained in the $\omega$-limit set of a recurrent critical point, then for all $\varepsilon>0$ there exists a neighbourhood $U$ of $x$ such that, for each $n \geqslant 0$ and each connected component $V^{\prime}$ of $f^{-n}(U)$,

(a) the spherical diameter of $V^{\prime}$ is $\leqslant \varepsilon$ and $\operatorname{deg}\left(f^{n}: V^{\prime} \longrightarrow U\right) \leqslant \delta$;

(b) for all $\varepsilon_{1}>0$ there exists $n_{0}>0$ such that if $n \geqslant n_{0}$, the spherical diameter of $V^{\prime}$ $i s \leqslant \varepsilon_{1}$.

The integer $\delta$ only depends on the degree of $f$.

We will say that an open set satisfies the $M(\varepsilon)$-condition if (a) and (b) of Theorem 3.2 are true for this set.

In the rest of this section, we assume that $f \in \mathrm{NR}(d)$, and we denote by $\mathscr{P}$ the set of parabolic points.

Lemma 3.3. For any $\varepsilon>0$, there is a finite covering $D_{i}$ of $J(f)$ such that

(C1) every $D_{i}$ is a round disk;

(C2) there exists $K \geqslant 1$ such that, for any $z \in J(f)$, there is an $i$ with

$$
\operatorname{Round}\left(D_{i}, z\right) \leqslant K \text {; }
$$

(C3) if $D_{i} \cap \mathscr{P}=\varnothing$, then $2 \cdot D_{i}$ satisfies the $M(\varepsilon)$-condition (where $2 \cdot D_{i}$ is the ball centred at the same point with doubled radius), and every connected component of $f^{-n}\left(D_{i}\right)$ contains at most one critical value;

(C4) for any $z \in J(f)$ which is not pre-parabolic, there are infinitely many iterates $n_{k} \geqslant 1$ such that $f^{n_{k}}(z)$ belongs to a $D_{i}$ satisfying (C2) and (C3).

Proof. Fix $\varepsilon>0$; we assume that $\varepsilon$ is smaller than the distance between any pair of critical values.

We will first define disk-neighbourhood $D(z)$ for every $z \in J(f)$. We choose sets covering the parabolic points; for any $z \in \mathscr{P}$, we pick pairwise disjoint neighbourhoods $U(z)$ of diameter smaller than $\varepsilon$ which contain at most one critical value at $\mathscr{P}$. For every $z \in \mathscr{P}$, we consider a disk $D(z) \subset f^{-1}(U(f(z))) \cap U(z)$ containing $z$. We name these sets 'parabolic domains'. In particular, for any $z \in J(f)$, if there is a time $n_{0} \geqslant 0$ such that for all $n \geqslant n_{0}$ the point $f^{n}(z)$ belongs to the parabolic domains, then $z$ is pre-parabolic.

For the other points, Theorem 3.2 allows us to choose round balls $D(z)$ so that (C1) and (C3) are satisfied.

The compactness of the Julia set allows us to extract a finite subcovering which still includes the sets chosen for $\mathscr{P}$ (this is actually automatic). Condition (C2) is satisfied since the covering is achieved by a finite number of sets.

If $z$ is not pre-parabolic, then there is a subsequence of iterates $\left(n_{k}\right)$ such that $f^{n_{k}}(z)$ is outside the parabolic domains; this means that $f^{n_{k}}(z) \in D_{i}$ for a certain disk of type (C2) and (C3). This proves (C4).

Proof of Proposition 3.1. Conditions (C1) and (C2) imply the existence of an $r>0$ such that, for any $z \in J(f)$, there is a disk $D_{i}$ of the covering such that $D(z, r) \subset D_{i}$.

Let $z \in J(f)$ be disjoint from the grand orbits of the parabolic points. It follows from condition (C4) that there are infinitely many iterates $f^{n_{k}}(z)$ such that 
$D\left(f^{n_{k}}(z), r\right)$ satisfies the $M(\varepsilon)$-condition. Let $N_{k}(z)$ be the connected component of $f^{-n_{k}}\left(D\left(f^{n_{k}}(z), r\right)\right)$ containing $z$. The $M(\varepsilon)$-condition implies that there is a degree $\delta>0$ such that $z \in G(\delta, r)$.

The proposition is now proved (the complement is countable and thus of zero length).

\section{Measure theoretic properties}

In this section, we prove the following theorem.

THEOREM 4.1. Let $f$ be weakly hyperbolic, then either $J(f)=\overline{\mathbb{C}}$, or $J(f)$ has measure zero.

Proof. Assume that $J(f)$ is not the whole sphere but has positive area, and that $f$ is normalized so that $J(f) \subset \mathbb{C}$. Let us consider a point of density $x$ of $J(f)$ which belongs also to a set $G(\delta, r)$. Then, for any $n \in E(x, D, r)$, one has

$$
\frac{\text { Area } D\left(f^{n}(x), r / 2\right) \backslash J(f)}{\text { Area } D\left(f^{n}(x), r / 2\right)} \asymp \frac{\operatorname{Area} B_{n}(x) \backslash J(f)}{\operatorname{Area} B_{n}(x)},
$$

where $B_{n}(x)$ is the connected component of $f^{-n} D\left(f^{n}(x), r / 2\right)$ containing $x$. Since $x$ is a point of density, the ratio on the right-hand side tends to 0 when $n \rightarrow \infty$. Therefore, by taking a limit $B$ of a subsequence of the balls $D\left(f^{n}(x), r / 2\right)$, we obtain that almost every point in $B$ belongs to $J(f)$; this implies that $J(f)$ contains an open set, and thus is the whole sphere.

Actually, for all the examples described in the previous section, an even stronger result holds: the Hausdorff dimension is strictly less than 2 (for topological Collet-Eckmann maps, this is proved in [19], for $\mathrm{NR}(d)$ this was proved by M. Urbański [26], and for maps satisfying the summability condition, the proof is given in [5]). We believe that this should be true for most uniformly weakly hyperbolic maps.

More generally, F. Przytycki has shown that, for any rational map, the Hausdorff dimension of the set of good points $\bigcup G(\delta, r)$ is the same as the so-called hyperbolic dimension of the map (see [18]). We thus deduce that, if a uniformly weakly hyperbolic map has a Julia set of dimension larger than 1, then it coincides with its hyperbolic dimension.

\section{Invariant line fields}

Most of this section is based on results due to C. McMullen which can be found in [13]. Let $X \subset \overline{\mathbb{C}}$. A line field supported on $X$ is given by a line $L_{x} \subset T_{x} \overline{\mathbb{C}}$ for almost every $x \in X$. Equivalently, we can define a line field by a Beltrami differential $\mu$ such that $|\mu|=1$ almost everywhere on $X$ and 0 elsewhere. A line field is said to be measurable if $\mu$ is measurable (see [13]).

Definition 5.1. Let $f$ be a rational map of degree $d \geqslant 2$. A measurable invariant line field is a measurable line field such that, for almost every $z \in \overline{\mathbb{C}}$,

$$
f^{*} L_{f(z)}=\left(T_{z} f\right)^{-1}\left(L_{f(z)}\right)=L_{z}
$$


These line fields are used to parametrize the quasiconformal deformations of rational maps (see [15]). Conjecturally, the only rational maps which carry an invariant line field on their Julia set are Lattès examples (cf. [11, 13, 15]).

Let $f$ be a rational map which carries an invariant line field $\mu$ on its Julia set. Without loss of generality (see [13, Chapter 2, §2.7]), we may assume that $\mu(0)=$ $d \bar{z} / d z$ and that 0 is a point of almost continuity, that is, for all $\varepsilon>0$,

$$
\lim _{r \rightarrow 0} \frac{\operatorname{Area}\{x \in D(0, r),|\mu(x)-\mu(0)|<\varepsilon\}}{\text { Area } D(0, r)}=1 .
$$

Proposition 5.2. In the above setting, if $0 \in G\left(\delta, r_{0}\right)$ for some $\left(\delta, r_{0}\right)$ then $f$ is a Lattès example.

Proof. By Proposition 2.3, there exist $\left(n_{k}\right), z_{k} \rightarrow 0$ and $\rho_{k} \rightarrow 0$ so that $g_{k}(z)=$ $f^{n}{ }_{k}\left(z_{k}+\rho_{k} z\right)$ tends to an open map $g$ with $\sup \left|z_{k}\right| / \rho_{k}<\infty$ and $g^{\prime}(0) \neq 0$. Let $r>0$ be small enough so that $\left.g\right|_{D(0,2 r)}$ is univalent. Then $g_{k}$ is univalent in $D(0, r)$ for $k$ large enough. Under these assumptions, C. McMullen has shown that there is a subsequence such that $g_{k}^{*} \mu$ tends to $d \bar{z} / d z$ on $D(0, r)$ [13, Theorem 5.16].

Let $g_{-1}: g(D(0, r)) \longrightarrow D(0, r)$ be its inverse. Then $\mu=g_{-1}^{*}(d \bar{z} / d z)$. Another result of C. McMullen then implies that $f$ is a Lattès example [13, Lemma 3.16].

An alternative proof is given by G. Martin and V. Mayer using Zalcman's lemma (see [17]); they actually show that the Zalcman limit map is the elliptic function which defines the Lattès example.

\section{Promoting topological conjugacies to quasiconformal maps}

In this section, we prove that a topological conjugacy has actually a better regularity on the Julia set by studying its metric properties. Let us recall that an orientation-preserving homeomorphism $\varphi$ of the plane is quasiconformal if there is a finite constant $H$ such that, for all $x \in \mathbb{C}$, one has

$$
\limsup _{r \rightarrow 0} \operatorname{Round}(\varphi(D(x, r)), \varphi(x)) \leqslant H .
$$

THEOREM 6.1 (J. Heinonen and P. Koskela). Let $\varphi: \mathbb{C} \longrightarrow \mathbb{C}$ be a homeomorphism such that there exists a constant $H<\infty$ for which the following is satisfied. For all $x \in \mathbb{C}$, there is a basis of simply connected neighbourhoods $U_{n}(x)$ such that

$$
\max \left\{\lim \sup \operatorname{Round}\left(U_{n}(x), x\right), \lim \sup \operatorname{Round}\left(\varphi\left(U_{n}(x)\right), \varphi(x)\right)\right\} \leqslant H .
$$

Then $\varphi$ is a quasiconformal map.

In their paper, J. Heinonen and P. Koskela actually prove this theorem for balls centred at $x$ [9], but they only use the fact that the volume of the balls is controlled by their diameter, which is still true for 'quasiballs' in the above sense.

Recently, this criterion has been improved by S. Kallunki and P. Koskela [10].

THeORem 6.2 (S. Kallunki and P. Koskela). In Theorem 6.1, one can allow an exceptional set $\mathscr{E}$ of at most $\sigma$-finite length on which no condition is required.

Our fundamental step is the following (compare with [20]). 
PROPOSITION 6.3. Let $f$ and $g$ be two rational maps conjugate by a homeomorphism $\varphi$. There is a constant $H=H(\varphi, \delta, r)$ such that, for all $x \in G(\delta, r)$,

$$
\max \left\{\lim \sup \operatorname{Round}\left(U_{n}(x), x\right), \lim \sup \operatorname{Round}\left(\varphi\left(U_{n}(x)\right), \varphi(x)\right)\right\} \leqslant H .
$$

Remark 6.4. Note that the property of belonging to some $G(\delta, r)$ is invariant under topological conjugacy up to a change of $r$.

Proof of Proposition 6.3. For all $n \in E(x, \delta, r)$, define $U_{n}(x)$ to be the connected component of $f^{-n}\left(D\left(f^{n}(x), r / 2\right)\right)$ which contains $x$. Then, diam $U_{n} \rightarrow 0$ and, by Theorem 1.4, we have Round $\left(U_{n}(x), x\right) \leqslant K$ for some $K$ independent of $n$. From the uniform continuity of $\varphi$ and $\varphi^{-1}$, all the sets $\varphi\left(D\left(f^{n}(x), r / 2\right)\right)$ have uniform roundness with respect to $f^{n}(x)$. Moreover, the moduli of

$$
\varphi\left(D\left(f^{n}(x), r\right)\right) \backslash \overline{\varphi\left(D\left(f^{n}(x), r / 2\right)\right)}
$$

are also uniformly bounded. Hence, we deduce that $V_{n}(x)$ has also uniform roundness with respect to $\varphi(x)$, where $V_{n}(x)$ is the connected component of $g^{-n} \circ \varphi\left(D\left(f^{n}(x), r / 2\right)\right)$ containing $\varphi(x)$, that is $V_{n}(x)=\varphi\left(U_{n}(x)\right)$.

Remark 6.5. F. Przytycki and S. Rohde were the first to use Theorem 6.1 to improve topological conjugacies. Moreover, Theorem 6.2 was motivated by a question of J. Graczyk and S. Smirnov for [5].

Proof of Theorem 0.2. (i) If $f$ is weakly hyperbolic, then the assumptions of Proposition 5.2 are satisfied.

(ii) From Proposition 6.3, the conjugacy satisfies Theorem 6.2, and thus, the map is quasiconformal. Moreover, if the Julia set is the whole sphere and if $f$ is not a Lattès example, then it follows from (i) that it does not carry any invariant line field, which implies that the conjugacy is globally conformal.

Proof of Corollary 0.3. Suppose now that $J(f) \neq \overline{\mathbb{C}}$ and $\infty \notin J(f)$. The first step is done as in [15] (see also [20]), that is we construct an isotopy rel $J(f)$ of $\varphi$ to get a new conjugacy $\varphi_{1}$ which is quasiconformal off $J(f)$. To do so, we distinguish two cases: Fatou basins for which the action of $f$ is discrete (corresponding to attracting and parabolic points) and the others.

For the first case, we can define an isotopy to a quasiconformal map on the quotient Riemann surfaces associated to these basins (see [15]), and then consider a lift compatible with $\varphi$ : thus, we obtain an isotopy $\varphi_{t}$ which conjugates $f$ to $g$ for all $t$. To see that it extends on the Julia set by $\varphi$, we use the fact that a neighbourhood of the boundary less the parabolic point (which attracts the points from the basin when it exists) is disjoint from the postcritical set; therefore, $\operatorname{diam} f^{-n}\left\{\varphi_{t}(x), 0 \leqslant\right.$ $t \leqslant 1$ \} tends to 0 as $n \rightarrow \infty$ (the inverse branches form a normal family, and the limits are in the Julia set, so they are constants).

For the second case, we have foliated disks split into annuli induced by the critical grand orbits. We can also define an isotopy to a quasiconformal map respecting the foliation. If the basin is super-attracting, then the same reasoning as above shows that this isotopy is rel the boundary of the basin. If the basin is a rotation domain (which seems to be unlikely) then its boundary has at most $\sigma$-finite length since no point can be in a good set. This means that the rotation extends continuously to the boundary; our map $\varphi$ is a rotation seen in linearizing coordinates, and an isotopy to a conformal map can be realized rel the boundary. 
Now Theorem 0.2 asserts that $\varphi_{1}$ is actually a globally quasiconformal map.

Acknowledgements. I would like to thank Tan Lei for being the motivation for this work, and S. Rohde and P. Koskela for fruitful discussions on Theorems 6.1 and 6.2. I would also like to thank K. Astala for many discussions about this work, and Tan Lei and Z. Balogh for their comments on a former version of this article. I am also grateful to the referee for his valuable comments.

\section{Appendix A. Parabolic points}

We are interested in germs of the form $g(z)=z+z^{v+1}+o\left(z^{v+1}\right)$ in a neighbourhood of the origin where $v \geqslant 1$ is an integer. We will show that, even though parabolic points are not good points for expansion, they are nonetheless not exceptional points in the sense of Theorem 6.2.

Proposition A.1. For any $\varepsilon>0$, there are neighbourhoods $N_{\varepsilon}$ and $N_{\varepsilon}^{\prime}$ of the origin and of infinity respectively, and there is a $(1+\varepsilon)$-quasiregular map $\psi_{\varepsilon}: N_{\varepsilon} \longrightarrow N_{\varepsilon}^{\prime}$ of degree $v$ such that, for all $z \in N_{\varepsilon}, \psi_{\varepsilon}(g(z))=\psi_{\varepsilon}(z)+1$ and whose restriction to the attracting petals is conformal. Moreover, $\psi_{\varepsilon}(z)=-1 /\left(v z^{v}\right)+O\left(1 / z^{v \alpha}\right)$ as $z \rightarrow 0$, for some $0<\alpha<1$.

Proof. Fix $\varepsilon>0$. We first define $\psi_{\varepsilon}$ on attracting petals by using Fatou coordinates. We now want to define an extension to the whole neighbourhood of the origin.

On any sector of opening $1 / v$, the change of variables $\zeta(z)=-1 /\left(v z^{v}\right)$ semiconjugates $g$ to a map $F(\zeta)=\zeta+1+O(1 / \zeta)$. Furthermore, for any $\eta>0$, the set $V_{\eta}$ of points such that $|F(\zeta)-(\zeta+1)|<\eta$ and $\left|F^{\prime}(\zeta)-1\right|<\eta$ is a neighbourhood of infinity.

We choose a sector bounded by two consecutive attracting axes. The map $F$ is well defined on a neighbourhood of infinity, except on $\mathbb{R}_{+}$. In this chart, Fatou coordinates have the following expansion: $\phi: \zeta \longmapsto \zeta(1+(a / \zeta) \log \zeta+O(1 / \zeta))$ as $\zeta$ tends to infinity in the set $\Sigma(R)=\{z, \operatorname{Re} z>R-k|\operatorname{Im} z|\}-\mathbb{R}_{+}$, where $R>0$ is large enough, and $0<k<1$ (see for example [24]).

Let $\eta>0$ be a small positive number whose value will be fixed later and will only depend on $\varepsilon$. Let $R_{\eta}>0$ be large enough so that $\left\{ \pm R_{\eta}\right\} \times \mathbb{R} \subset V_{\eta}$, and $\phi$ is $\eta$-close to identity on $\Sigma\left(R_{\eta}\right)$. We will now work with its inverse $\chi$.

For any $t \in \mathbb{R}$, define $\mathscr{L}_{t}$ to be the maximal $F$-forward invariant line generated by $\chi([R, R+1] \times\{t\})$.

There exists $t_{\eta}>0$ so that $\mathscr{L}_{ \pm t_{\eta}} \subset V_{\eta /\left(2 R_{\eta}\right)}$ and is parametrized by the real line with constant speed. We can thus extend $\chi$ on both half-planes $\left\{|\operatorname{Im} z|>t_{\eta}\right\}$. Moreover, the chain rule implies that

$$
\left|\chi\left(-R_{\eta} \pm i t_{\eta}\right)-\left(-R_{\eta} \pm i t_{\eta}\right)\right| \leqslant \eta
$$

Let $Q(\eta)$ be the quadrilateral bounded by $\chi\left(\left[-R_{\eta}-1,-R_{\eta}\right] \times\left\{ \pm t_{\eta}\right\}\right)$, $\left[\chi\left(-R_{\eta}-1-i t_{\eta}\right), \chi\left(-R_{\eta}-1+i t_{\eta}\right)\right]$ and its image by $F$. If $\eta$ is small enough, then $Q(\eta)$ is well defined.

We want to define a homeomorphic extension $\chi:\left[-R_{\eta}-1,-R_{\eta}\right] \times\left[-t_{\eta}, t_{\eta}\right] \longrightarrow$ $Q(\eta)$ which conjugates the translation to $F$ on the vertical sides. By construction, $\chi$ is close to the identity on the horizontal sides for the $\mathscr{C}^{1}$ norm. This means that there 
is a quasiconformal extension close to the identity, hence with small dilatation ratio. We can also extend $\chi$ on the left half-space using the functional equation. If $\eta$ is chosen small enough, then the map $\chi$ is $(1+\varepsilon)$-quasiconformal.

The conformality of $\psi_{\varepsilon}$ at the origin follows from the fact that the origin is a measurable deep point (see [14, Theorem 2.25, and example 4 thereafter]).

Definition A.2. Let $g(z)=z+z^{v+1}+o\left(z^{v+1}\right)$ and let $\psi$ be the map constructed in Proposition A.1, we define the canonical basis of neighbourhoods $N_{R}, R \geqslant R_{0}$, of 0 with respect to $\psi$ to be the preimages by $\psi$ of the complement of the squares $[-R, R] \times[-R, R]$ for $R$ large enough.

Lemma A.3. Let $g(z)=z+z^{v+1}+o\left(z^{v+1}\right)$.

(a) Let $N_{R}, R \geqslant R_{0}$, be a canonical basis of neighbourhoods of 0 . Then their roundness with respect to the origin is uniformly bounded.

(b) Let $\varphi$ be a topological conjugacy with another parabolic germ $g_{1}$. If the restriction of the homeomorphism to the attracting petals is conformal, then the images by $\varphi$ of $N_{R}$ are also uniformly 'quasiround'.

Proof. (a) From Corollary 1.7, the sets $N_{R}$ have bounded roundness.

(b) Let $N_{R}^{\prime}=\varphi\left(N_{R}\right)$. Since $\varphi$ is conformal on the attracting petals, we can alter $\varphi$ in the repelling petals to get a new map $\varphi_{1}$ so that $\psi_{1}=\psi \circ \varphi_{1}^{-1}$ satisfy the same properties as $\psi$. Let us consider $\psi_{1}\left(N_{R}^{\prime}\right)$; these sets agree with $\psi\left(N_{R}\right)$ except on a fixed strip. By rescaling these sets by $\zeta \longmapsto\left(R_{0} / R\right) \zeta$, we see that, as $R \rightarrow \infty$, they converge for the Hausdorff metric of compact sets to $\psi\left(N_{R_{0}}\right)$. It follows that they have bounded roundness, and this in turn implies bounded roundness for $N_{R}^{\prime}$.

\section{References}

1. L. V. AhLFors, Lectures on quasiconformal mappings (Van Nostrand, 1966).

2. D. Bargman, 'Simple proofs of some fundamental properties of the Julia set', Ergodic Theory Dynam. Systems 19 (1999) 553-558.

3. F. Berteloot and J. Duval, 'Une démonstration directe de la densité des cycles répulsifs dans l'ensemble de Julia', Progress in Mathematics 188 (Birkhauser, 2000) 221-222.

4. L. Carleson, P. Jones and J. C. Yoccoz, 'Julia \& John', Bol. Soc. Brasil. Mat. 25 (1994) 1-30.

5. J. GRACZYK and S. SMIRnOv, 'Weak expansion' (in preparation).

6. P. HaïssinSKY, 'Pincement de polynômes', preprint 235, ENS de Lyon, 1999 (revised version).

7. P. HAÏSSINSKY and L. TAN, 'Mating and pinching' (in preparation).

8. W. Hayman, Multivalent functions (Cambridge University Press, 1958).

9. J. Heinonen and P. Koskela, 'Definitions of quasiconformality', Invent. Math. 120 (1995) 61-79.

10. S. Kallunki and P. Koskela, 'Exceptional sets for the definition of quasiconformality', Amer. J. Math. 122 (2000) 735-743.

11. S. Lattès, 'Sur l'itération des substitutions rationnelles et les fonctions de Poincaré', C. R. Acad. Sci. Paris 166 (1918) 26-28.

12. M. LyUbich and Y. Minsky, 'Laminations in holomorphic dynamics', J. Differential Geom. 47 (1997) $17-94$.

13. C. T. MCMullen, 'Complex dynamics and renormalization', Annals of Mathematics Studies 135 (Princeton University Press, Princeton, NJ, 1994).

14. C. T. McMullen, 'Renormalization and 3-manifolds which fiber over the circle', Annals of Mathematics Studies 142 (Princeton University Press, Princeton, NJ, 1996).

15. C. T. MCMullen and D. Sullivan, 'Quasiconformal homeomorphisms and dynamics. III: The Teichmüller space of a holomorphic dynamical system', Adv. Math. 135 (1998) 351-395.

16. R. MAÑ́, 'On a theorem of Fatou', Bol. Soc. Brasil. Mat. 24 (1993) 1-11.

17. G. MARTIN and V. MAYER, 'Rigidity in holomorphic and quasiregular dynamics', preprint, 1999.

18. F. PrZYTYCKI, 'Conical limit sets and Poincaré exponent for iterations of rational functions', Trans. Amer. Math. Soc. 351 (1999) 2081-2099.

19. F. Przytycki and S. RohDE, 'Porosity of Collet-Eckmann Julia sets', Fund. Math. 155 (1998) 189-199. 
20. F. Przytycki and S. Rohde, 'Rigidity of holomorphic Collet-Eckmann repellers', Ark. Mat. 37 (1999) 357-371.

21. F. Przytycki and M. Urbański, 'Rigidity of tame rational functions', Bull. Polish Acad. Sci. Math. 47 (1999) 163-182.

22. W. Schwick, 'Repelling periodic points in the Julia set', Bull. London Math. Soc. 29 (1997) 314-316.

23. H. Shiga, 'On complex analytic properties of limit sets and Julia sets', preprint, 1999.

24. M. Shishikura, 'The parabolic bifurcations of rational maps', Colóquio Brasileiro de Matemática 19 (1993).

25. M. SHISHIKURA and L. TAN, 'An alternative proof of Mañé's theorem on non-expanding Julia sets', The Mandelbrot set, theme and variations, London Mathematical Society Lecture Note Series 274 (ed. L. Tan, Cambridge University Press, 2000) 265-279.

26. M. URbański, 'Rational functions with no recurrent critical points', Ergodic Theory Dynam. Systems 14 (1994) 391-414.

27. L. Zalcman, 'Normal families: new perspectives', Bull. Amer. Math. Soc. 35 (1998) 215-230.

\author{
Mathematisches Institut \\ University of Bern \\ Sidlerstrasse 5 \\ CH-3012 Bern \\ Switzerland
}

phaissin@math-stat.unibe.ch 